\title{
Gestión del compromiso, prácticas laborales que la fortalecen. Caso de estudio
}

\author{
Commitment management, labor practices strengthening it. Study case
}

Doi: 10.22458/rna.v1 1i 2.2646

Ing. Stefanya Leonela Arias Pérez

Universidad Tecnológica Equinoccial, Ecuador

slap-995@hotmail.com

https://orcid.org/0000-0002-4570-6272
Mag. Héctor López Paredes

Universidad Internacional del Ecuador, Ecuador

helopezpa@uide.edu.ec

https://orcid.org/0000-0003-0456-5271

\section{RESUMEN}

El propósito de este trabajo es analizar ¿Cuáles son las prácticas de talento humano (TTHH) que tienen una relación positiva con la gestión del compromiso? tomando como caso de estudio una empresa de tecnología de la ciudad de Quito - Ecuador. Se empleó el cuestionario como instrumento para la recolección de información y el análisis correlacional para responder la pregunta de investigación. Los resultados permiten identificar los factores que influyen de manera directa y positiva en el compromiso de los colaboradores con la organización y sus objetivos, evidenciando la influencia de la gestión de talento humano como un componente estratégico.

\begin{abstract}
The purpose of this work is to analyze the kinds of human talent practices that have a positive relation on commitment management based on the study case of a technological company in Quito, Ecuador. A questionnaire was used as an instrument to gather information and make a correlational analysis to answer the research question. The results enable to identify the factors that have a direct and positive influence on the collaborators' commitment to the organization and its objectives while evidencing the influence of human talent management as a strategic component.
\end{abstract}

\section{RÉSUMÉ}

Le but de ce travail est d'analyser les types de pratiques de talent humain qui ont une relation positive sur la gestion des engagements sur base du sujet d'étude d'une entreprise technologique dans la ville deQuito, Équateur. Un questionnaire a été utilisé comme instrument de collecte d'informations et d'analyse corrélationnelle afin de répondre à la question de la recherche. Les résultats permettent d'identifier les facteurs qui influencent de manière directe et positive les engagements des collaborateurs envers l'organisation et ses objectifs, en mettant en évidence l'influence de la gestion du talent humain comme composante stratégique.

\section{RESUMO}

O objetivo deste trabalho é analisar quais são as práticas de talento humano (TTHH) que têm uma relação positiva com a gestão do compromisso?, tendo como estudo de caso uma empresa de tecnologia da cidade de Quito - Equador. O questionário foi utilizado como instrumento de coleta de informação e análise correlacional para responder à questão de investigação. Os resultados permitem identificar os fatores que influenciam de maneira direta e positivamente no compromisso dos colaboradores com a organização e os seus objetivos, evidenciando a influência da gestão do talento humano como componente estratégico.

\section{PALABRAS CLAVE: \\ COMPROMISO \\ ORGANIZACIONAL, GESTIÓN DE TALENTO \\ HUMANO, BUENAS \\ PRÁCTICAS DE TALENTO \\ HUMANO, GESTIÓN DEL \\ COMPROMISO.}
KEY WORDS:
ORGANIZATIONAL
COMMITMENT, HUMAN
TALENT MANAGEMENT,
GOOD PRACTICES
OF HUMAN TALENT,
COMMITMENT
MANAGEMENT.

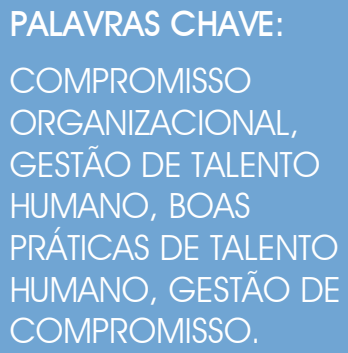

PALAVRAS CHAVE:

COMPROMISSO

ORGANIZACIONAL, GESTÃO DE TALENTO HUMANO, BOAS PRÁTICAS DE TALENTO HUMANO, GESTÃO DE COMPROMISSO.
MOTS CLÉS:

\section{ENGAGEMENT} ORGANISATIONNEL, GESTION DU TALENT HUMAIN, BONNES PRATIQUES DU TALENT HUMAIN, GESTION DES ENGAGEMENTS. 


\section{INTRODUCCIÓN}

Harter et al. (2002) consideran que "el compromiso laboral incluye al denominado involucramiento de los individuos y la satisfacción y entusiasmo por el trabajo", por tanto, el compromiso organizacional es el apego afectivo o emocional que tiene el colaborador hacia su organización en los diferentes factores: sentirse identificado con la organización en relación con metas, creencias, ideas etc. (Gatewood, R. y Riordan, C. 1997), sentir que es parte de la organización y finalmente fidelización y lealtad hacia la organización provocando defenderla como propia Claure y Bohrt (2015). Cuando existe un cambio organizacional, el diagnóstico es fundamental, este debe estar enfocado en conocer cultura, objetivos y esquemas organizacionales que permitan tener su identidad vigente, todo esto para aumentar la motivación, el compromiso y tener un clima de trabajo más agradable. Como lo menciona Rodríguez (2015), el personal requiere de tiempo para comprender, ajustarse e involucrarse al cambio, mostrándose como un aspecto positivo mediante la comunicación y confianza bilateral.

En la actualidad, el compromiso de los colaboradores con la organización constituye un activo importante (Vandenbergue, C.; Bentein, K., 2009), puede ser uno de los mecanismos que tiene la dirección de talento humano para analizar la lealtad y vinculación con su organización (Sandoval Duque, J. L., 2014), siendo importante para las organizaciones conocer no sólo el tipo, sino también el grado de compromiso de sus miembros (Golik, M., 2013), por ello, los debates actuales evidencian los problemas en el cumplimiento de resultados en las organizaciones, atribuidas a la falta de compromiso de los colaboradores con los propósitos estratégicos, siendo para Pardo y Porras (2011) necesario trabajar en la participación de la gestión del talento humano en el éxito de la organización, de esta manera, dicha gestión es vista como un conjunto de acciones tendientes a demostrar el nivel de relación causal entre el impacto de diversos programas y políticas de talento humano y los estados financieros de la empresa.

El presente trabajo considera importante destacar el papel fundamental del área de Talento humano en el diseño de los sistemas de gestión y procesos que ayudan a la ejecución de la estrategia de negocio (Gatewood, R. y Riordan, C., 1997), mediante la alineación de las funciones del área tales como: el reclutamiento, la contratación, la capacitación y desarrollo, la administración de beneficios y la planificación de la sucesión, con los objetivos organizacionales Héctor Macías, Nayeli Escobar (2013), reconociendo la relevancia de conocer a los colaboradores y saber ubicarlos estratégicamente, para un mayor desempeño y productividad, así como, desde la visión del colaborador el cumplimiento de su necesidad de sentirse útil, por ello, los resultados obtenidos, evidencian como (1) el compromiso afectivo del trabajador hacia la organización se relaciona a las variables de planes de desarrollo y carrera que estimulen la productividad y calidad en el trabajo; (2) la imagen corporativa se relaciona de manera positiva con el compromiso de los colaboradores cuando sienten que su trabajo brinda un aporte a la comunidad; (3) respecto al clima organizacional y conectividad si la empresa alinea los objetivos organizacionales con el trabajo genera importancia en el desarrollo de sus funciones y transmite empoderamiento mediante una comunicación efectiva.

\section{Revisión de literatura}

Meyer y Allen (1991), quienes definen al compromiso como "un estado psicológico que caracteriza la relación del colaborador con la organización, el cual tiene implicaciones para la decisión de continuar siendo parte de la misma" para estos autores, las características de las personas, las experiencias de socialización, las condiciones ambientales y las prácticas de talento humano son consideradas como variables antecedentes distales que impactan el desarrollo del compromiso con la organización, señalando algunos autores como (Netemeyer et al., 1996; Grandey et al., 2005) que puede generar conflicto en el trabajo, disminuyendo así la relación familiar y acarreando problemas que terminan afectando a la empresa directa e indirectamente. 
Lo que genera compromiso, empoderamiento e identidad corporativa en los colaboradores parte de la influencia que ejercen los líderes en sus equipos de trabajo (Harter et al., 2002), este se convierte en puntos claves de la empresa, reconocido como capital humano, el cual es un activo más flexible, a través de él se puede implementar, reacomodar, adaptar y hacer factible cualquier adelanto tecnológico, concepto teórico o aplicación práctica, lo que le otorga mayor vitalidad a las organizaciones Pardo (2007), siendo fundamental en este contexto la influencia que ejerce el tipo de liderazgo como línea de supervisión (Vandenbergue, C.; Bentein, K., 2009).

El compromiso organizacional se ha convertido en un tema de fundamental importancia en relación con la productividad y actitudes de los colaboradores en la organización, respecto a los resultados que se han obtenido a partir de lograr este (Antón y González, 2000). Macías y Escobar (2013) mencionan que la atracción y retención del talento deberían ser prácticas complementarias que se alineen a la estrategia de la organización y a la estructura orgánico-funcional de su empresa, el punto clave para la atracción de talento es entender qué actividades se pueden aplicar para lograr un balance de la vida laboral y personal, que permita generar flexibilidad en el trabajo a sus colaboradores y al mismo tiempo alcanzar los objetivos planteados, las brechas generacionales han producido diferencias notables en las preferencias de los incentivos, siendo los factores de motivación diferente para cada uno.

Según Whitener (2001), los colaboradores vinculan las tareas y prácticas de gestión de talento humano como señales del compromiso de la organización y muestran reciprocidad, mejorando la percepción sobre su propio compromiso con la organización. Dorenbosch, Van Engen y Verhagen (2005) hallaron evidencias de una relación positiva entre las prácticas de talento humano orientadas al compromiso y el sentimiento de propiedad del trabajo por parte de los colaboradores, siendo según Pons y Ramos (2012) necesario identificar las prácticas de Talento Humano que generen compromiso, aumenten la productividad y mejoren el ambiente laboral como una de las tareas del responsable de gestionar al recurso humano, se toma en cuenta el tamaño, giro de negocio, posibilidades y necesidades de la empresa para alcanzar los objetivos planteados alineados a la organización, entre las generales encontramos:

\section{La gestión del compromiso como una propuesta de valor competitiva}

La compensación es una práctica por medio de la cual se evalúa la contribución de los colaboradores en la empresa mediante el cumplimiento de sus funciones para establecer recompensas que sean monetarias y no monetarias, directas e indirectas, de acuerdo con las normas laborales vigentes y con las políticas propias de la organización Dolan, Schuler y Valle (1999), una adecuada estrategia de compensación apoyará en la alineación y dirección del esfuerzo de los colaboradores hacia el logro de los objetivos estratégicos, recompensando y mejorando el desempeño individual y de equipo, para generar un mayor sentido de pertenencia, estimulando la colaboración y compromiso, incrementando la productividad, así como la optimización de recursos Macías y Escobar (2013), por supuesto se debe considerar la relación hacia las necesidades del colaborador y posibilidades de la organización, que permitan el desarrollo y mejora en el desempeño del colaborador y como resultado la organización atraiga y retenga el talento que necesita. La perspectiva estratégica de las compensaciones tiene relación con la retribución recibida por los colaboradores que permita aumentar la motivación y el desarrollo de las personas, así también se plantean acciones para fortalecer los objetivos, la filosofía y la cultura organizacional de la empresa, (Calderón y Álvarez, 2006; Richard y López, 2020).

En las organizaciones, los planes de desarrollo de personal como las promociones internas se toman en el marco de sistemas formal o informal Grueso H. (2010). Los sistemas formales de promoción se caracterizan porque han sido definidos y desarrollados formalmente a partir de un claro establecimiento de trayectorias profesionales por parte de la organización Gómez, Balkin y Cardy (2001); en contraste, los sistemas informales de promoción de personal no interponen criterios preestablecidos, se caracterizan por ser manejados con unas ases ad hoc y, debido a la ausencia de un procedimiento sistemático y estructurado, cada caso de promoción se analiza y decide de manera aislada Powell (1999). 
La $4^{\text {a }}$ Encuesta de CEO en México (2013) mencionada por Macías y Escobar (2013), el 69\% a nivel global expusieron estar dispuestos a responder a ofertas agresivas que la competencia hace a sus ejecutivos talentosos para mantenerlos en sus organizaciones. Cuando la empresa está planeando las carreras profesionales de sus colaboradores es más probable que sean ellos mismos quien se fijen metas profesionales y trabajen activamente por obtenerlas (Calderón y Álvarez, 2006). Según Werther y Davis (2000) las empresas que promueven el plan de carrera obtienen ventajas en el desarrollo de los colaboradores con potencial, en la disminución de la tasa de rotación de personal y más éxito en las promociones internas.

Entre las funciones de talento humano está la retención de personal, generar estabilidad y confianza en los colaboradores, ofrecer alternativas de crecimiento y desarrollo que estimulen la productividad y calidad en el trabajo que desempeñan (Golik, M., 2013), entre las mejores prácticas que proponen Héctor Macías, Nayeli Escobar (2013) se encuentran:

- Planes de desarrollo y carrera

- Planes de sucesión y continuidad del negocio

- Coaching y Mentoring ejecutivo

El desempeño define el rendimiento laboral, es decir, la capacidad de una persona para producir, hacer, elaborar, acabar y generar trabajo en menos tiempo, con menor esfuerzo y mejor calidad, estando dirigido a la evaluación la cual dará como resultado su desenvolvimiento. El desempeño laboral es la eficacia del personal que trabaja dentro de las organizaciones, funcionando la persona con una gran labor y satisfacción laboral (Chiavenato, 2002, citado en Bonifacio y Falconi, 2016).

Para Chiavenato (2000), la evaluación del desempeño constituye una técnica de dirección imprescindible en la actividad administrativa. Es un medio que permite localizar problemas de supervisión de personal, integración del empleado a la organización o al cargo que ocupa en la actualidad, desacuerdos, desaprovechamiento de empleados con potencial más elevado al requerido por el cargo, motivación, etc.

Por otro lado, Quispe (2015) señala que, la productividad, eficacia y eficiencia laboral son dimensiones del desempeño laboral.

\section{Reconstruir confianza}

La $4^{\text {a }}$ Encuesta de CEO en México (2013) señaló que el 56\% de los CEO tienen como principal prioridad para los próximos 12 meses, enfocarse en el marco del clima y la cultura organizacional Macías y Escobar (2013).

Incentivar la participación de los colaboradores crea una empresa sólida, capaz de desarrollar colaboradores que aporten al desempeño de la organización, crear una cultura de confianza y apertura a ideas innovadoras y mejora continua, que mantenga un clima y cultura organizacional saludable para el desempeño de las funciones. Esto en definitiva es un ganar-ganar para la empresa, mientras incrementa el bienestar e impulsa una cultura de confianza, eleva el compromiso en los colaboradores e incrementa la productividad en el mercado, como menciona Macías y Escobar (2013), la correcta implementación de buenas prácticas puede permitir desarrollar estilos de liderazgo y una cultura que mantenga en alto los valores de la organización y su desempeño, incluso en momentos críticos, en donde se propone:

1) Fomentar una práctica participativa, donde todos los agentes relevantes colaboran en el proceso y luego apoyan las decisiones tomadas, establece una cultura organizacional ganadora.

2) Prácticas como el establecimiento de un tablero de indicadores, los programas wellness o incluso la creación de comités orientados al mantenimiento del clima organizacional, pueden ser detonadores de niveles de efectividad que contribuyan al desarrollo del capital humano en una organización. 
La generación Millenials, que en la actualidad domina el sector laboral, se caracteriza por la búsqueda de una conexión emocional hacia su profesión, esta generación pretende encontrar algo más allá de una buena oportunidad laboral para desempeñar su carrera profesional, esperan además encontrar un ambiente laboral y prácticas que contribuyan con su calidad de vida Héctor Macías, Nayeli Escobar (2013) como:

1) Balance de vida personal y trabajo, que incorpora elementos tanto personales como laborales que atribuyen a dicho balance entre estos las horas trabajadas, las demandas de la dirección de la empresa, las expectativas familiares, el número de hijos o la situación conyugal Eby et al. (2005). Por un lado, el rol que cumple el colaborador en la empresa demanda un gran compromiso con la organización, lo cual se traduce en ocasiones en una mayor expectativa del Gerente de que sus colaboradores deben pasar largas jornadas en el trabajo y priorizar su vida laboral a su vida privada Hughes y Bozionelos (2007). De existir un conflicto de relación vida personal y trabajo Sánchez, Cegarra, y Cegarra (2011) propone estas tres variables dependientes que se deben a su carácter relevante a nivel empresarial: (1) El deseo de abandonar la empresa como variable fundamental que está relacionada directamente con la tasa de rotación o abandono de los colaboradores de la empresa; (2) El desempeño de los colaboradores que recoge el grado en que los colaboradores de forma individual cumplen con las exigencias y tareas asociadas al puesto de trabajo, calidad y cantidad de trabajo realizado y nivel adecuado de productividad; (3) El compromiso de los colaboradores o compromiso actitudinal, debido a que se espera que tenga importantes efectos sobre el comportamiento de los colaboradores en las empresas Vandenbergue y Bentein (2009). Twenge et al. (2010), señala que los valores relacionados al tiempo libre y al ocio, es decir, a la vida personal y familiar, se incrementan frecuentemente a través de las generaciones mientras que el interés específico de la realización de sus funciones en el trabajo decae.

2) Impacto en la carga de trabajo, relacionada al interés de explorar y encontrar respuestas a las expectativas de la generación Y adquiere relevante importancia en las empresas, las organizaciones deben ingeniarse estrategias para atraer a los jóvenes de alto potencial y lograr así una ventaja competitiva Batt y Valcour (2003); De Cieri et al. (2005). Como consecuencia, entre las características principales de este grupo encontramos que quieren tener éxito profesional, buscan un trabajo interesante que les brinde variedad, responsabilidad y desafío; es decir, valoran el significado en el trabajo Arnett (2004).

3) Manejabilidad de la carga de trabajo, con esto se refiere a la necesidad de acuerdos flexibles de trabajo, que les permitan además de cumplir con su trabajo atender su vida privada Jerkiewicz (2000). Rechazan la invasión de la vida privada por el trabajo, según la literatura, a diferencia de la generación anterior, donde las variables económicas eran dominantes, hoy se valoran otros beneficios, tales como flexibilidad temporal y espacial y entornos de trabajo informales Bosco y Bianco (2005), a pesar de que saben que tendrán que trabajar más de 40 horas semanales, valoran mucho más el tiempo destinado a actividades personales y familiares, trabajar con el modelo del cumplimiento de objetivos que les permita distribuir su tiempo en las diferentes metas establecidas, coordinando con los objetivos personales, genera flexibilidad en su espacio y eleva el desempeño Lewis et al. (2002).

\section{Generar compromiso laboral, desarrollo y oportunidades}

En la actualidad, generaciones como los millenials se caracterizan por ser profesionales con potencial, altamente preparados, empapados de todo lo referente a tecnología y con una mentalidad disruptiva, los aspectos que demandan de la empresa es transparencia y participación en la toma de decisiones (Sandoval Duque, J. L., 2014), no les interesan las jerarquías y creen en un sistema basado en la conciliación y la búsqueda de la felicidad Martín (2015). Brindarle a un Millenial la confianza y autonomía en la toma de decisiones aporta a su motivación, darle retos constantemente hace que su trabajo no caiga en monotonía o rutina y se sienta útil para la organización. La firma de consultoría en Talento humano Manpower Group, identificó 10 rasgos que caracterizan a esta generación, uno de ellos explicaba que en el ámbito laboral les gusta ser incluidos en la toma de decisiones importantes, así como, saber que están aportando un valor agregado a la empresa Rodríguez, Terán y García (2016). Esta generación también busca apoyo para el desarrollo profesional, para ser formados y guiados a lo largo de su carrera profesional por los líderes de las organizaciones Rodríguez et al. (2016), al ser una generación que busca siempre cambios continuos, es importante tener un plan de carrera definido y que la empresa le brinde las herramientas necesarias para el cumplimiento de este plan y que sientan el acompañamiento de su jefe. 
Finalmente, es importante destacar el papel fundamental del área de Talento humano en el diseño de los sistemas de gestión del talento y procesos que ayudan a la ejecución de la estrategia de negocio de la organización, lograrán este objetivo mediante la alineación de las funciones del área, tales como el reclutamiento, la contratación, la capacitación y desarrollo, la administración de beneficios y la planificación de la sucesión, con los objetivos de negocio que son primordiales. Asimismo, se lanzan programas, políticas y algunos proyectos que se consolidan como elementos clave en la estrategia de talento humano de una organización Héctor Macías, Nayeli Escobar (2013). Es fundamental en el área de talento humano conocer a los colaboradores y saber ubicarlos estratégicamente, es positivo tanto para la empresa como para el colaborador, la empresa conseguirá resultados en el desempeño y productividad mientras que el colaborador al sentirse útil en la organización y cómodo en las tareas que realiza (alineación puesto/persona) sentirá que el aporte que hace es una retribución a la empresa (Villacrés y López, 2018). Las organizaciones que aplican buenas prácticas en su estrategia se vuelven parte integral del éxito de la organización Macías y Escobar (2013) considerando 4 pilares fundamentales:

Cultura: Fomentar comportamientos que reflejen la visión y estrategia organizacional.

Capacidad: Enfrentar los cambiantes requerimientos de los clientes y de la propia organización.

Conectividad: Asegurar la cercanía de la organización a sus clientes a través del uso de redes sociales.

Costo: Alinear los costos y el desempeño para aprovechar los recursos financieros.

\section{METODOLOGÍA DE LA INVESTIGACIÓN}

Para esta investigación, se utilizó el nivel de estudio correlacional, donde se relacionó las variables de buenas prácticas en el departamento de talento humano y compromiso laboral, para conocer cómo se pueden comportar si existiera la variable número uno en relación con la variable número dos, es decir, si el compromiso laboral depende de la aplicación correcta de las buenas prácticas en el departamento de talento humano, empleando el método inductivo deductivo para analizar los resultados a partir de la información y situaciones que se recolectaron en la investigación.

\section{Tabla 1}

Distribución de la población

\begin{tabular}{lcc} 
ÁREA & $\begin{array}{c}\text { NÚMERO DE } \\
\text { COLABORADORES }\end{array}$ & $\%$ \\
\hline Administrativa & 15 & $30 \%$ \\
\hline Comercial & 12 & $24 \%$ \\
\hline Servicios & 23 & $46 \%$ \\
\hline TOTAL & 50 & $100 \%$ \\
\hline
\end{tabular}

Fuente: elaboración propia. 


\section{Población y muestra.}

La población objeto de estudio son los colaboradores de Quito y Guayaquil comprendidos entre los 22 y los 68 años de edad, que están en los 3 principales departamentos de la empresa, como resultado se obtiene una población total de 50 personas en el año 2018 - 2019, distribuidos de la siguiente manera (ver tabla 3):

Debido al tamaño de la población finita que se ha establecido se realiza una prueba piloto que según Malhotra (2004) la define como la aplicación de un cuestionario a una pequeña muestra de encuestados para identificar y eliminar los posibles problemas de la elaboración del cuestionario.

\section{Selección de instrumentos.}

Se definió la encuesta como instrumento de investigación con el objetivo de obtener la información lo más acertada a la realidad en opiniones y hechos determinados del estudio. Para el análisis de la primera variable referente al compromiso laborar, se tomó el instrumento de compromiso de Meyer, Allen y Smith (1993) que evalúa los tres tipos de compromisos mencionados en la presente investigación en el marco teórico: afectivo, normativo y de continuidad, este instrumento fue aplicado en un estudio realizado en la Pontificia Universidad Católica del Ecuador (PUCE), realizando una modificación que permitió adaptar las preguntas a la empresa en estudio Hidrovo y Espín (2016), consta de 6 ítems sumando un total de 18 preguntas debidamente clasificadas de la siguiente manera: del 1 al 6 se encuentran las preguntas correspondientes al compromiso afectivo, del 7 al 12 las del compromiso de continuidad y del 13 al 18 las del compromiso normativo, el formato de calificación Likert se estableció desde 5 (totalmente de acuerdo) hasta 1 (totalmente en desacuerdo).

En la segunda parte del instrumento, se construyó las preguntas para evaluar la segunda variable referente a buenas prácticas de Talento Humano, el formato de calificación para las preguntas emplea una escala de Likert desde 5 (totalmente de acuerdo) hasta 1 (totalmente en desacuerdo), tomando en cuenta los factores mencionados en el marco teórico de la presente investigación referente a buenas prácticas de Talento Humano. Para correr las encuestas a los colaboradores de la empresa de tecnología, se aplicó la prueba de fiabilidad del coeficiente Alfa de Cronbach en el programa estadístico SPSS versión 22 a fin de conocer los elementos que se deberían tomar en cuenta para medir la fiabilidad en la aplicación del instrumento.

\section{Procesamiento de datos.}

Para la recolección de la información, se realizó una explicación breve de los cuestionarios por aplicarse en los colaboradores de forma introductoria, se aplicó a 44 colaboradores, los cuales decidieron participar de forma voluntaria, los datos obtenidos fueron tabulados mediante la herramienta de SPSS que permitió realizar el análisis de correlación para determinar la relación entre las variables obtenidas y el grado de asociación entre las mismas, como cita Enriquez (2019) a LONG (1966) "El análisis de correlación estudia el grado de asociación de dos o más variables."

Para el procesamiento de información se procedió en primer lugar a ordenar los datos por área para facilitar el proceso de tabulación, ingresando la información en una base; en segundo lugar, se realizó la tabulación en el sistema SPSS, el cual ayudó a obtener el análisis de correlación de Pearson que según Levin y Rubin (1996) es un índice que mide el grado de variación existente de las dos variables en relación; en tercer lugar, se analizó e interpretó los resultados. 


\section{Tabla 2}

Correlación entre compromiso laboral y buenas prácticas de talento humano

\begin{tabular}{|c|c|c|c|c|c|c|c|c|c|c|c|c|c|}
\hline \multicolumn{14}{|c|}{ Buenas prácticas de TTHH } \\
\hline & & \multicolumn{4}{|c|}{$\begin{array}{l}\text { Ofrecer una } \\
\text { propuesta de } \\
\text { valor competitiva }\end{array}$} & \multicolumn{3}{|c|}{$\begin{array}{l}\text { Desarrollar talento } \\
\text { desde adentro }\end{array}$} & \multirow{2}{*}{$\begin{array}{c}\text { Alinear El Talento } \\
\text { Humano A La } \\
\text { Estrategia } \\
\text { Organizacional } \\
\\
\text { Clima } \\
\text { Organizacional } \\
\text { y conectividad }\end{array}$} & \multicolumn{2}{|c|}{$\begin{array}{l}\text { Reconstruir } \\
\text { la Confianza }\end{array}$} & \multicolumn{2}{|c|}{$\begin{array}{l}\text { Equilibrio vida } \\
\text { personal y laboral }\end{array}$} \\
\hline & & 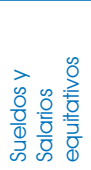 & 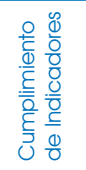 & 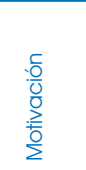 & $\begin{array}{l}\stackrel{0}{0} \\
\frac{0}{0} \\
\frac{0}{0} \\
\frac{D}{\Phi} \\
\infty\end{array}$ & 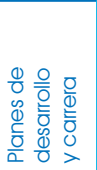 & 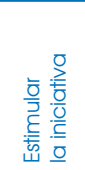 & $\begin{array}{l}\frac{1}{0} \\
\frac{0}{0} \\
\frac{0}{0} \\
0 \\
\frac{0}{0} \\
u\end{array}$ & & $\begin{array}{l}\text { Participación } \\
\text { de los } \\
\text { colaboradores } \\
\text { en la empresa }\end{array}$ & $\begin{array}{l}\text { Imagen } \\
\text { Corporativa }\end{array}$ & $\begin{array}{l}\text { Calidad } \\
\text { de vida }\end{array}$ & $\begin{array}{c}\text { Flexibilidad } \\
\text { de horarios } \\
\text { y vida } \\
\text { personal }\end{array}$ \\
\hline \multirow{6}{*}{ 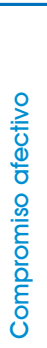 } & $\begin{array}{l}\text { Correlación } \\
\text { de Pearson }\end{array}$ & 0,231 & 0,457 & 0,282 & 0,389 & 0,594 & 0,397 & 0,364 & 0,427 & 0,460 & 0,703 & 0,473 & 0,450 \\
\hline & $\mathrm{N}$ & 44 & 44 & 44 & 44 & 44 & 44 & 44 & 44 & 44 & 44 & 44 & 44 \\
\hline & $\begin{array}{l}\text { Correlación } \\
\text { de Pearson }\end{array}$ & 0,219 & 0,379 & 0,142 & 0,398 & 0,555 & 0,423 & 0,379 & 0,424 & 0,410 & 0,542 & 0,414 & 0,382 \\
\hline & $\mathrm{N}$ & 44 & 44 & 44 & 44 & 44 & 44 & 44 & 44 & 44 & 44 & 44 & 44 \\
\hline & $\begin{array}{l}\text { Correlación } \\
\text { de Pearson }\end{array}$ & 0,228 & 0,374 & 0,054 & 0,471 & 0,629 & 0,418 & 0,326 & 0,528 & 0,506 & 0,652 & 0,364 & 0,368 \\
\hline & $\mathrm{N}$ & 44 & 44 & 44 & 44 & 44 & 44 & 44 & 44 & 44 & 44 & 44 & 44 \\
\hline \multirow{6}{*}{ 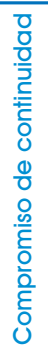 } & $\begin{array}{l}\text { Correlación } \\
\text { de Pearson }\end{array}$ & 0,292 & 0,320 & 0,327 & 0,279 & 0,440 & 0,494 & 0,342 & 0,278 & 0,427 & 0,389 & 0,312 & 0,319 \\
\hline & $\mathrm{N}$ & 44 & 44 & 44 & 44 & 44 & 44 & 44 & 44 & 44 & 44 & 44 & 44 \\
\hline & $\begin{array}{l}\text { Correlación } \\
\text { de Pearson }\end{array}$ & 0,282 & 0,183 & 0,318 & 0,296 & 0,421 & 0,441 & 0,365 & 0,203 & 0,342 & 0,398 & 0,382 & 0,307 \\
\hline & $\mathrm{N}$ & 44 & 44 & 44 & 44 & 44 & 44 & 44 & 44 & 44 & 44 & 44 & 44 \\
\hline & $\begin{array}{l}\text { Correlación } \\
\text { de Pearson }\end{array}$ & 0,208 & 0,217 & 0,249 & 0,237 & 0,380 & 0,464 & 0,307 & 0,232 & 0,241 & 0,382 & 0,262 & 0,290 \\
\hline & $\mathrm{N}$ & 44 & 44 & 44 & 44 & 44 & 44 & 44 & 44 & 44 & 44 & 44 & 44 \\
\hline \multirow{6}{*}{ 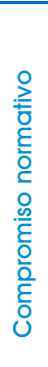 } & $\begin{array}{l}\text { Correlación } \\
\text { de Pearson }\end{array}$ & 0,228 & 0,318 & 0,274 & 0,432 & 0,402 & 0,473 & 0,491 & 0,523 & 0,411 & 0,530 & 0,366 & 0,428 \\
\hline & $\mathrm{N}$ & 44 & 44 & 44 & 44 & 44 & 44 & 44 & 44 & 44 & 44 & 44 & 44 \\
\hline & $\begin{array}{l}\text { Correlación } \\
\text { de Pearson }\end{array}$ & 0,299 & 0,350 & 0,209 & 0,451 & 0,386 & 0,375 & 0,516 & 0,390 & 0,428 & 0,472 & 0,312 & 0,344 \\
\hline & $\mathrm{N}$ & 44 & 44 & 44 & 44 & 44 & 44 & 44 & 44 & 44 & 44 & 44 & 44 \\
\hline & $\begin{array}{l}\text { Correlación } \\
\text { de Pearson }\end{array}$ & 0,286 & 0,253 & 0,169 & 0,519 & 0,377 & 0,340 & 0,444 & 0,319 & 0,443 & 0,388 & 0,429 & 0,304 \\
\hline & $\mathrm{N}$ & 44 & 44 & 44 & 44 & 44 & 44 & 44 & 44 & 44 & 44 & 44 & 44 \\
\hline
\end{tabular}

Correlación mínima

Correlación baja

Correlación moderada

Fuente: elaboración propia. 


\section{RESULTADOS}

Entendiendo que las variables son características o cualidades que se presentan o no en las personas o grupos de personas y en modalidades diferentes (Bernal, 2010), en el presente estudio se consideran a los elementos detectados de las buenas prácticas de Talento Humano y las tres clases de compromiso propuestas por Meyer y Cols (1993), se registraron los siguientes resultados (Ver tabla 2)

\section{Tabla 3}

Correlaciones significativas

\begin{tabular}{|c|c|c|c|c|c|c|c|c|}
\hline & & & \multirow{2}{*}{$\begin{array}{l}\text { Ofrecer una } \\
\text { propuesta de } \\
\text { valor competitiva } \\
\\
\text { Beneficios }\end{array}$} & \multicolumn{2}{|c|}{$\begin{array}{l}\text { Desarrollar talento } \\
\text { desde adentro }\end{array}$} & \multirow{2}{*}{$\begin{array}{c}\text { Alinear El Talento } \\
\begin{array}{c}\text { Humano a la Estrategia } \\
\text { Organizacional }\end{array} \\
\begin{array}{c}\text { Clima Organizacional } \\
\text { y conectividad }\end{array}\end{array}$} & \multicolumn{2}{|c|}{$\begin{array}{l}\text { Reconstruir la } \\
\text { Confianza }\end{array}$} \\
\hline & & & & $\begin{array}{l}\text { Planes de } \\
\text { desarrollo } \\
\text { y carrera }\end{array}$ & Capacitación & & $\begin{array}{l}\text { Participación de } \\
\text { los colaboradores } \\
\text { en la empresa }\end{array}$ & $\begin{array}{l}\text { Imagen } \\
\text { Corporativa }\end{array}$ \\
\hline \multirow{12}{*}{ 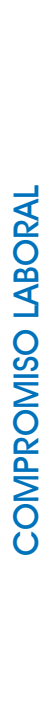 } & \multirow{6}{*}{ 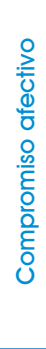 } & $\begin{array}{l}\text { Correlación } \\
\text { de Pearson }\end{array}$ & $N / A$ & 0,594 & $\mathrm{~N} / \mathrm{A}$ & $\mathrm{N} / \mathrm{A}$ & $N / A$ & $0,703^{* *}$ \\
\hline & & $\mathrm{N}$ & $\mathrm{N} / \mathrm{A}$ & 44 & $\mathrm{~N} / \mathrm{A}$ & N/A & $N / A$ & 44 \\
\hline & & $\begin{array}{l}\text { Correlación } \\
\text { de Pearson }\end{array}$ & $\mathrm{N} / \mathrm{A}$ & 0,555 & $\mathrm{~N} / \mathrm{A}$ & $\mathrm{N} / \mathrm{A}$ & $\mathrm{N} / \mathrm{A}$ & 0,542 \\
\hline & & $\mathrm{N}$ & $\mathrm{N} / \mathrm{A}$ & 44 & $\mathrm{~N} / \mathrm{A}$ & $N / A$ & $N / A$ & 44 \\
\hline & & $\begin{array}{l}\text { Correlación } \\
\text { de Pearson }\end{array}$ & $\mathrm{N} / \mathrm{A}$ & $0,629 * *$ & $\mathrm{~N} / \mathrm{A}$ & 0,528 & 0,506 & $0,652^{\star \star}$ \\
\hline & & $\mathrm{N}$ & $N / A$ & $\mathrm{~N} / \mathrm{A}$ & $\mathrm{N} / \mathrm{A}$ & 44 & 44 & 44 \\
\hline & \multirow{6}{*}{ 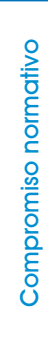 } & $\begin{array}{l}\text { Correlación } \\
\text { de Pearson }\end{array}$ & N/A & N/A & N/A & 0,523 & $\mathrm{~N} / \mathrm{A}$ & 0,530 \\
\hline & & $\mathrm{N}$ & $N / A$ & N/A & N/A & 44 & $\mathrm{~N} / \mathrm{A}$ & 44 \\
\hline & & $\begin{array}{l}\text { Correlación } \\
\text { de Pearson }\end{array}$ & $\mathrm{N} / \mathrm{A}$ & $\mathrm{N} / \mathrm{A}$ & 0,516 & $N / A$ & $\mathrm{~N} / \mathrm{A}$ & $\mathrm{N} / \mathrm{A}$ \\
\hline & & $\mathrm{N}$ & $\mathrm{N} / \mathrm{A}$ & $\mathrm{N} / \mathrm{A}$ & 44 & $N / A$ & $N / A$ & $\mathrm{~N} / \mathrm{A}$ \\
\hline & & $\begin{array}{l}\text { Correlación } \\
\text { de Pearson }\end{array}$ & 0,519 & $\mathrm{~N} / \mathrm{A}$ & N/A & N/A & N/A & N/A \\
\hline & & $\mathrm{N}$ & N/A & $\mathrm{N} / \mathrm{A}$ & $\mathrm{N} / \mathrm{A}$ & N/A & $N / A$ & $\mathrm{~N} / \mathrm{A}$ \\
\hline
\end{tabular}

Fuente: elaboración propia. 


\section{Análisis Correlacional}

Para el análisis correlacional de los datos, se tomaron los valores significativos superiores a 0.6 correspondientes a correlación buena y correlación muy buena (ver tabla 2) de las variables del compromiso laboral con las buenas prácticas de talento humano, excluyendo los valores cuya correlación de Pearson es menor a 0.4 correspondientes a correlación baja y mínima, dicho esto se arrojan los datos con valores significativos:

Las correlaciones expresadas en la tabla 3, muestran el factor compromiso afectivo con una relación positiva de 0.629 con el desarrollo de planes carrera, evidenciando que la empresa a pesar de ser pequeña ofrece alternativas de crecimiento y desarrollo que estimulen la productividad y calidad en el trabajo que desempeñan, desarrollando lealtad e identificando a las personas con los objetivos empresariales. Otro resultado importante es una correlación moderada de 0.594 y 0.555 donde la felicidad, lealtad y fidelización de los colaboradores, también, se crea mediante el ascenso o promoción en la organización mediante el factor desempeño, entendiendo de acuerdo a (Martín, 2015; Rodríguez Garza et al., 2016; Richard y López, 2020) que la falta de planes de crecimiento y desarrollo disminuyen la lealtad referente al compromiso afectivo en el cual la persona establece lazos emocionales intensos con su organización.

El resultado obtenido en relación de la imagen corporativa analizada en las buenas prácticas de TTHH y el compromiso afectivo es de 0.703 de correlación buena, el cual se refiere a que los colaboradores son felices en la empresa si esta genera valor a la comunidad (Golik, M., 2013; Martín, 2015), como resultado de una estrategia efectiva de Responsabilidad Social (Héctor Macías, Nayeli Escobar, 2013). Los resultados respaldan el planteamiento de Claure, M., y Bohrt, M. (2015), respecto que los colaboradores generaran un compromiso afectivo en la organización cuando sientan que su trabajo brinda un aporte a la comunidad, de no ser así este disminuye (Pardo y Porras, 2011).

En el aspecto capacitación arroja una correlación de 0.516 en relación con el compromiso normativo, evidenciado en los planes y programas de capacitación individuales que, según Héctor Macías, Nayeli Escobar, A. R. (2013) van de acuerdo a las necesidades específicas del cargo, permitiendo desarrollar sentimientos de obligación del colaborador y un sentido del deber moral de continuar perteneciendo a la organización (Ríos Manríquez, M., Del, M., Téllez Ramírez, R., y Ferrer Guerra, J., 2010). Dicho de otra manera, el no capacitar a los colaboradores para el correcto desempeño de sus tareas, genera en los colaboradores el deseo de abandonar la empresa al no sentirse con las herramientas necesarias en su trabajo (Claure, M., y Bohrt, M., 2015).

Respecto al clima organizacional y conectividad tiene una correlación moderada de 0.528 en relación con el compromiso afectivo, entendiendo que al alinear los objetivos organizacionales con el trabajo de los colaboradores se genera importancia en el desarrollo de sus funciones y transmite empoderamiento (Ríos Manríquez, M., Del, M., Téllez Ramírez, R., y Ferrer Guerra, J., 2010). En referencia al clima organizacional y conectividad existe una correlación moderada de 0.523 con el compromiso normativo, que se refiere al ambiente laboral que existe en la empresa, este contribuye al compromiso laboral de los colaboradores y al mismo tiempo crea compromiso por los beneficios que reciben (Héctor Macías, Nayeli Escobar, A. R., 2013). La participación de los colaboradores en la empresa tiene una correlación moderada de 0.506 con respecto del compromiso afectivo, donde se debe considerar que los objetivos de la organización hagan importante el trabajo de sus colaboradores (Claure, M., y Bohrt, M., 2015; Richard y López, 2020).

Los resultados muestran correlaciones moderadas pertenecientes a beneficios y compromiso normativo que es de 0.519, es decir, los colaboradores en la empresa muestran deseo de permanecer en la empresa siempre y cuando sientan que los beneficios que esta les brinda cubren sus necesidades (Merlin Patricia Grueso H., 2010; Ríos Manríquez, M., Del, M., Téllez Ramírez, R., y Ferrer Guerra, J., 2010; Sandoval Duque, J. L., 2014), debiendo orientar las prácticas de talento humano para fortalecer esta relación en favor de generar estabilidad laboral para el cumplimiento de actividades y metas (Claure, M., y Bohrt, M., 2015). 
Existe una correlación buena de 0.652 referente a imagen corporativa y compromiso afectivo, donde los colaboradores consideran que al trabajar en la empresa generan valor a la comunidad, creando un ambiente familiar que permite su desarrollo en la sociedad, siendo para Héctor Macías, Nayeli Escobar, A. R. (2013) importante orientar prácticas de talento humano que permitan alinear los propositos individuales a los colectivos y organizacionales, así como, generar valor a la comunidad crea lealtad y fidelización en los colaboradores (Sandoval Duque, J. L., 2014).

\section{CONCLUSIONES}

Una vez revisados y analizados los datos obtenidos por medio del cuestionario aplicado a la empresa de tecnología, se deprenden resultados que muestran la relevancia del presente estudio en la generación de prácticas de talento humano que permitan alinear los esfuerzos individuales a los colectivos y organizacionales, siendo los planes de desarrollo y carrera con un 0.629 de correlación, la imagen corporativa con 0.703 y 0.652 de correlaciones prácticas del compromiso afectivo que permiten incrementar valor (Sandoval Duque, J. L., 2014), elevar el sentido de pertencia (Golik, M., 2013; Claure, M., y Bohrt, M., 2015) y mejorar las condiciones de desarrollo profesional para las personas (Rodríguez Garza et al., 2016)

Los resultados muestran la importancia de la construcción de modelos de beneficios e incentivos, capacitación, clima organizacional, conectividad participación de los colaboradores en la empresa con resultados sobre el 0.5 de correlación, que con base en las actuales prácticas de Talento Humano analizadas en la empresa de tecnología motivo de estudio se deben mejorar, a efecto de buscar una mayor influencia del liderazgo en el clima organizacional (Pons Verdú, F. J., y Ramos López, J., 2012; Villacrés y López, 2018), el desarrollo de espacios de comunicación que permitan un mayor entendimiento y desarrollo del compromiso afectivo (Claure, M., y Bohrt, M., 2015) así como, la apertura de espacios para una participación activa de los colaboradores en las mejoras tanto laborales como de los resultados y metas planteados (Rodríguez Garza et al., 2016).

De las variables analizadas en el marco teórico que generan compromiso en los colaboradores de la empresa de tecnología se encuentran: ofrecer una propuesta de valor competitiva (Sandoval Duque, J. L., 2014), desarrollar talento desde adentro (Claure, M., y Bohrt, M., 2015), alinear el talento humano a la estrategia organizacional (Héctor Macías, Nayeli Escobar, A. R., 2013), reconstruir la confianza y equilibrio vida personal y laboral (Rodríguez Garza et al., 2016). Los resultados muestran la importancia de orientar prácticas de talento humano que permitan reconstruir la confianza y desarrollar talento desde adentro con referencia al compromiso afectivo (Héctor Macías, Nayeli Escobar, A. R., 2013; Richard y López, 2020), destacando el enfoque en imagen corporativa y planes de desarrollo y carrera, los mismos que se indican como parte de la propuesta (Sandoval Duque, J. L., 2014; Richard y López, 2020). 


\section{REFERENCIAS}

Antón, C. y González, l. (2000). evidencia empírica de la naturaleza multidimensional del compromiso con la organización. Arnett, J. (2004). Emerging adulthood. New York: Oxford University Press.

Batt, R. y Valcour, P. (2003) Human resources practices as predictors of work-family outcomes and employee turnover. Industrial Relations, 42 (2), 189-196.

Calderón, G., y Álvarez, C. (2006). Características y sentido. 42(142), 26-45.

Claure, M., y Bohrt, M. (2015). Tres Dimensiones Del Compromiso Organizacional: Identificación, Membresía Y Lealtad. Ajayu Órgano de Difusión Científica Del Departamento de Psicología UCBSP, 1991, 7.

Cuenca, R. y López, H. (2020). Estudio de la gestión del compromiso y cultura organizacional de FLACSO Rotation of Public Managers. Problem of management or solution of political trust? 37, 43-56. https://doi.org/10.31095/podium.202

Dorenbosch, L., Van Engen, M., y Verhagen, M. (2005). On- the-job Innovation: The Impact of Job Design and Human Resource Management through Production Ownership. Creativity and Innovation Management, 14, 129-141

Dolan, S.; Schuler, R. y Valle, R. (1999). La gestión de los Recursos Humanos. Madrid: McGraw Hill

Eby, L.T.; Casper, W.J.; Lockwood, A.; Bordeaux, C.; Brinley, A. (2005) "Work and fam- ily research in IO/OB: Content analysis and review of the literature", Journal of Vocational Behavior, Vol. 66, p. 124-197.

Gatewood, R. y Riordan, C. (1997). The development and test of a model of total quality: Organizational practices, TQ principles, employee attitudes and customer satisfaction. Journal of Quality Management, 2(1), 41-65.

Gómez Mejía, L. R.; Balkin, D. y Cardy, R. (2001). Dirección y gestión de Recursos Humanos (3a. ed.). Madrid: Prentice Hall.

Grandey, A.; Cordeiro, B.L.; Crouter, A.C. (2005), A longitudinal and multisource test of the work-family conflict and job satisfaction, Journal of Occupational and Organizational Psychology, Vol., 78, p. 305-323.

Grueso, P. (2010). Implementación de buenas prácticas de promoción de personal y su relación con su cultura y el compromiso con la organización. 20, 79.90.

Harter J., Schmidt F., Hayes T., (2002). Bussines unit level relationship between employee satisfaction, employee engagement and bussines outcomes a meta-analysis.

Hughes, J.; Bozionelos, N. (2007), Work life balance as source of job dissatisfaction and withdrawal attitudes. An exploratory study on the views of male workers

Jones, Bosco, S. y Bianco, C. (2005). Influence of mater- nal work patterns and socio-economic estatus on Gen Y lifestyle choice. Journal of Career Deve- lopment, 32, 165-182.

Lewis, S.; Smithson, J. y Kugelberg, C. (2002). In- to work: job insecurity and changing psychological contracts. In J. Brannen, S. Lewis, A. Nilsen and J. Smithson (Eds.), Young Europeans, work and family, 69-88, London: Routledge.

LONG, R. A. (1966). Analisis Correlación y regresión. Journal of Accountancy, 121(4), 84-85.

Macías y Escobar, A. R. (2013). Recursos Humanos People \& Change. Mejores Prácticas En Recursos Humanos, 1.12.

Martín, A. (2015). Horizonte 2020 ¿Esperanza o advertencia? Revista Estudios de Juventud. 108(6) 9-20. ISSN: 0211-4364. Obtenido de: http://www.injuve.es/sites/default/files/2015/39/publicaciones/Revista_108.pdf

Meyer, J., Smith, C. y Allen, N. (1993). Commitment to organizations and occupations: extension and test of a threecomponent conceptualization. Journal of Applied Psychology, 78(4), 538-551.

Meyer J.P., Allen N.J., (1991). A three component conceptualization of organizational commitment, Human Resource Management Review 1, 61-98

Netemeyer, R. G.; J. S. Boles; R. McMurrian (1996), Development and Validation of Work- Family Conflict and FamilyWork Conflict Scales, Journal of Applied Psychology, Vol. 81, núm. 4, p.400-410. 
Pardo, C. (2007). Gestión del talento humano basado en competencias, la clave para lograr el éxito empresarial [tesis de maestría]. Bogotá: Universidad de La Salle.

Pardo, C., y Porras, J. (2011). La gestión del talento humano ante el desafío de organizaciones competitivas. Gestión \& Sociedad, 4(2), 167-183.

Pons Verdú, F. J., y Ramos López, J. (2012). Influencia de los Estilos de Liderazgo y las Prácticas de Gestión de RRHH sobre el Clima Organizacional de Innovación Influence of Leadership Styles and Human Resources Management Practices on Innovation Climate in Organizations. Revista de Psicología Del Trabajo y de Las Organizaciones, 28(2), 81-98. https://doi.org/10.5093/tr2012a7

Powell, G. (1999). Reflections on the glass ceiling: Recent trends and future prospects.

Rodríguez-Garza, B. N., Terán-Cázares, M. M., y García-Dela Peña, M. E. (2016). Factores que influyen en el trabajo colaborativo de la generación Millennials: Estudio de alumnos universitarios en México. VinculaTégica, 3(1).

Rodríguez, D. (2015). Diagnóstico organizacional SupleEEMer \Clase Ejecutiva. 2015.

Sánchez, E., Cegarra, D., y Cegarra, J. (2011). ¿Influye el conflicto trabajo-vida personal de los empleados en la empresa? Does the Work to Life Conflict Affect the Organization? Universia Business Review.

Twenge, J., Campbell, S., Hoffman, B. y Lance, C. (2010). Generational differences in work values: leisure and extrinsic values increasing, social and intrinsic values decreasing. Journal of Management, 36 (5), 1117-1142.

Vandenbergue, C.; Bentein, K. (2009) A closer look at the relationship between affective commitment to supervisors and organizations and turnover, Journal of Occupational and Organizational Psychology, Vol. 82, p. 331-348

Villacrés, J., y López, H. (2018). INCIDENCIA DEL TIPO DE LIDERAZGO EN EL CLIMA LABORAL CASO: ÁREA DE CAJAS DE LAS AGENCIAS DE QUITO DE UNA ENTIDAD FINANCIERA. Economía y Negocios, 9. https://doi.org/https://doi.org/10.29019/eyn.v9i1.429

Werther, W. B. Jr. y Davis, K (2000) Dirección de Personal y Recursos Humanos. México: McGraw Hill

Whitener, E. M. (2001). Do 'high commitment' human resource practices affect employee commitment? Journal of Management, 27, 515-535. 